\title{
Violencia de Género en el Trabajo en Chile. Un Campo de Estudio Ignorado
}

\section{GENDER VIOLENCE AT WORK IN CHILE. AN IGNORED FIELD OF STUDY}

\author{
Ximena Díaz Berr', Amalia Mauro Cardarelli', Elisa Ansoleaga Moreno², Juan Pablo Toro Cifuentes ${ }^{3}$ \\ 1. Investigadora Centro de Estudios de la Mujer, Chile \\ 2. Directora Escuela de Psicología y académica del Programa de Estudios Psicosociales del Trabajo, Universidad Diego Portales, Chile \\ 3. Académico Programa de Estudios Psicosociales del Trabajo, Universidad Diego Portales, Chile
}

\begin{abstract}
RESUMEN
Este artículo expone resultados del estudio "Dimensiones organizacionales de la violencia en el trabajo en Chile considerando desigualdades ocupacionales y de género" (Fondecyt Regular 2014. № 1140060). Se focalizó en tres sectores económicos: industria del retail, bancaria y de elaboración de alimentos. Para cada uno se seleccionó una muestra no probabilística de tres actores laborales: trabajadores y trabajadoras, directivos y jefaturas, y dirigentes/as sindicales. Se aplicó una metodología cualitativa basada en entrevistas en profundidad. Los resultados indican que la violencia en el trabajo deriva de relaciones laborales caracterizadas por un fuerte desbalance de poder entre ejecutivos y trabajadores/as, y por el predominio de estilos de supervisión autoritarios que buscan disciplinar a la fuerza de trabajo. Se enmarca en el concepto de "régimen de trabajo opresivo". La violencia de género en el trabajo expresa la articulación de dos formas de opresión sobre las mujeres: su bajo poder formal en las organizaciones y su bajo poder social derivado de relaciones de género que las subordinan y discriminan. Se manifiesta en mayor exposición al maltrato asociado a ocupaciones de menor estatus y a condiciones precarias de empleo, en actitudes y conductas "machistas", acoso sexual y discriminaciones asociadas a la maternidad.
\end{abstract}

(Díaz X, Mauro A, Ansoleaga E, Toro J, 2017. Violencia de Género en el Trabajo en Chile. Un Campo de Estudio Ignorado. Cienc Trab. EneAbr; 19 [58]: 42-48).

Palabras Claves: VIOLENCIA EN EL TRABAJO, DESBALANCE DE PODER, INEQUIDADES DE GÉNERO.

\section{ABSTRACT}

This article presents results of the study "Organizational dimensions of violence at work in Chile considering occupational and gender inequalities" (Fondecyt Regular 2014. No. 1140060). It focused on three economic sectors: retail, banking and food processing. For each one, a non-probabilistic sample of three labor actors was selected: workers, managers and leaders, and union leaders.

A qualitative methodology based on in-depth interviews was applied. The results indicate that violence at work derives from labor relations characterized by a strong imbalance of power between executives and workers, and by the predominance of authoritarian supervision styles that seek to discipline the labor force. It is part of the concept of "oppressive work regime"

Gender violence at work expresses the articulation of two forms of oppression against women: their low formal power in organizations and their low social power derived from gender relations that subordinate and discriminate against them. It manifests itself in greater exposure to ill-treatment associated with occupations of lower status and precarious employment conditions, "macho" attitudes and behaviors, sexual harassment and discrimination associated with motherhood.

Key words: WORKPLACE VIOLENCE, POWER IMBALANCE, GENDER INEQUALITIES

\section{INTRODUCCIÓN}

La violencia en el trabajo es reconocido en todo el mundo como uno de los problemas sociales emergentes que mayor preocupación provoca por su creciente magnitud y consecuencias a nivel personal, organizacional y social. ${ }^{1}$ En Chile, estudios de carácter exploratorio advierten que la violencia se ha instalado en forma

\section{Correspondencia / Correspondence:}

Ximena Díaz Berr

Paseo Bulnes 120, oficina 88, Santiago Centro, Chile

e-mail:ximenadb@gmail.com

Recibido: 23 de Diciembre de 2016 / Aceptado: 25 de Febrero de 2017 permanente en las relaciones laborales y que afecta más a las mujeres. ${ }^{2-7}$ Sin embargo, se ha indagado muy poco en factores explicativos. Particularmente importante es una encuesta a una muestra nacional de trabajadores y trabajadoras asalariados/as en el país realizada el año 2011 que reveló una importante prevalencia de violencia en el trabajo y diferencias significativas de exposición según el sexo. ${ }^{8}$ Este estudio, asimismo, adelanta algunas asociaciones entre violencia laboral y factores de riesgo psicosocial. Un primer problema que se enfrenta cuando se aborda el estudio de la violencia laboral es que el concepto de violencia es tan amplio que los límites de lo que es considerado aceptable o no son a menudo muy vagos y la percepción de lo que constituye violencia en diferentes contextos y culturas es tan diversa, que proponer una definición constituye un desafío. Una de las definiciones más aceptadas señala que estamos ante una conducta de violencia "cuando uno o varios individuos perciben 
persistentemente en un periodo de tiempo que reciben acciones negativas de una o varias personas, en una situación donde el/la sujeto acosado/a tiene dificultades para defenderse contra esas acciones". ${ }^{9-10}$ La definición enfatiza la persistencia y duración de la exposición tanto como la existencia de diferencias de poder entre perpetradores y víctimas. Un conflicto no es violencia si el incidente es aislado y si las dos partes tienen una fuerza relativamente similar en el conflicto. Esta dimensión referida a desbalance de poder entre víctimas y victimarios es considerada una condición determinante de la violencia, reflejando tanto las desigualdades derivadas de la estructura de poder formal de las organizaciones como también de otras formas potenciales de poder social. ${ }^{11-14}$ Los y las trabajadores/as con bajo poder social son más vulnerables a diversas formas de abusos y son, a menudo, quienes ocupan posiciones con bajo poder formal en las organizaciones, como ocupaciones de bajo estatus en la jerarquía organizacional. ${ }^{11,15}$ Una fuente de poder social es el género. El género se enmarca en relaciones sociales de poder definidas por la lógica del patriarcado y se imponen mediante normas y valores culturales y simbólicos. ${ }^{16}$ "Impregna además las instituciones sociales porque no solo se refiere a las relaciones entre los sexos en el ámbito individual, sino también a una gama compleja de estructuras, prácticas y comportamientos que definen los sistemas de organización que constituyen las sociedades humanas. Por lo tanto, se trata de un principio organizador de la vida social". ${ }^{17}$ Ahora bien, la desigual distribución del poder y las relaciones asimétricas que se establecen entre varones y mujeres en nuestra sociedad están directamente vinculadas a la violencia de género18 que se diferencia de otras formas de agresión en que el factor de riesgo o de vulnerabilidad es el solo hecho de ser mujer. En este artículo esperamos aportar reflexiones que contribuyan a comprender de qué modo las desigualdades de poder basadas en el género, interactúan con desigualdades de poder en las relaciones laborales, determinando una mayor vulnerabilidad de las mujeres a la violencia en los espacios de trabajo. Es necesario señalar que el artículo se centrará solo en la violencia que se ejerce sobre las mujeres, dejando por el momento de lado la violencia que se ejerce sobre minorias sexuales, que de acuerdo a los testimonios escuchados constituye un problema necesario de ser abordado en profundidad.

\section{MÉTODO}

\section{Muestra}

El estudio se focalizó en tres sectores económicos -industria del retail, bancaria y de elaboración de alimentos- que han experimentado importantes transformaciones asociadas a la modernización, globalización y desregulación económica y laboral en Chile. ${ }^{19}$ Para cada una de ellas, se seleccionó una muestra no probabilística de tres actores laborales: i) trabajadores hombres y mujeres asalariados/as, con antigüedad laboral de al menos dos años, con una jornada de trabajo de al menos 30 horas semanales, y estar directamente contratado/a por la empresa. ii) hombres y mujeres en cargos de jefaturas intermedias o gerencias, y iii) dirigentes y dirigentas sindicales. El proceso muestral se orientó por los principios de saturación teórica y una lógica de muestreo secuencial conceptualmente conducido. La muestra quedó constituida por 70 personas: 34 hombres y 36 mujeres, distribuidas de la siguiente manera: 7 gerentes (4 mujeres), 12 jefaturas intermedias (5 mujeres), 35 trabajadores (22 mujeres) y 11 representantes sindicales (5mujeres).

\section{Método}

Se utilizó una metodología cualitativa de investigación basada en entrevistas en profundidad orientadas a recoger las percepciones de los casos seleccionados sobre: a) conductas, actitudes, incidentes, que se consideran violencia en el trabajo; b) tipos de violencia más recurrentes; b) dimensiones organizacionales que favorecen o inhiben las manifestaciones de violencia; c) personas o grupos más expuestos a violencia, d) quiénes las ejercen, y e) formas de enfrentamiento, entre los temas más relevantes que fueron abordados. La información recogida fue analizada, codificada y traspasada al programa ATLAS-ti. Los y las entrevistados/as fueron informados/as del objetivo del estudio, garantizándoles las condiciones de voluntariedad, anonimato, y confidencialidad absoluta en el manejo de los datos.

\section{RESULTADOS}

\section{Antecedentes del contexto: relaciones laborales autocrá- ticas en Chile}

En la percepción de los y las trabajadores entrevistados, la violencia laboral es expresión de un modelo de relaciones laborales signado por el verticalismo y por el predominio de estilos de liderazgo y de supervisión autoritarios, que se trasmiten desde las más altas gerencias hasta los/as trabajadores/as en los puestos de menor poder, pasando por todos los mandos intermedios. Algunos asimilan este tipo de liderazgo a la figura de "patrón de fundo".

Me tocó ver cuando el gerente general, dueño de la empresa, trató a garabatos a un gerente adelante mio. Yo lo miré y dije 'de qué estamos hablando', y el gerente prácticamente agachó la cabeza. Es la politica del patrón de fundo que ya en este país está arraigada (Dirigente sindical, industria del retail)

Por otra parte, sus testimonios señalaron que la violencia se manifiesta a través de una amplitud de formas que incluyen gritos, amenazas, descalificaciones, que es una violencia sostenida y que se dirige en general al colectivo o grupos de trabajadores/as, más que a un/a trabajador/a en particular. Un dirigente de la industria de alimentos indica, por ejemplo, que los supervisores “(...) te gritan encima de toda la gente, te reclaman en grupo, yo eso lo encuentro una falta de respeto, porque siempre tendrian que llamarte a un lugar donde puedan conversar contigo, conversar no gritarte", percepción compartida por una dirigenta del retail que dice que "los jefes presionan con el tono de voz, es mucho más alto, muy, muy autoritarios y duros, duros en la forma de la expresión, y generalmente aplicando descalificaciones".

Esta percepción de violencia generalizada hace referencia al concepto de "régimen de trabajo opresivo", donde el grupo es el objetivo, más que al concepto de victimización donde un individuo es el objetivo, y que cuando es así, probablemente ocurre en un contexto opresivo. ${ }^{10}$ Esta forma de violencia busca disciplinar a la fuerza de trabajo, adaptarla a las normas y exigencias de las empresas, de producción, de jornadas u otras. Las necesidades de los/as trabajadores/as no importan.

(...) a ellos (jefaturas) no les interesa el trabajador, no les interesa, solamente les interesa que el trabajador le produzca y si yo no le produzco porque estoy medio resfriada, me tengo que ir para mi 
casa, porque no sirvo, ¿me entiende? (Trabajadora, industria de alimentos)

Este rasgo de las empresas chilenas ha sido destacado por Ramos ${ }^{20}$ quien ha señalado que si bien han ocurrido cambios positivos en las empresas desde los años 70, en materia de relaciones internas siguen prevaleciendo relaciones verticales con un alto diferencial de poder y donde existe un marcado grado de desconfianza y distancia social entre ejecutivos y trabajadores. Por su parte Rodríguez y Gómez ${ }^{21}$ destacan, por una parte, la existencia de una gran presión ejercida por los gerentes y niveles superiores enfocados en la eficiencia organizacional, y por otra, la inexistencia de autonomía y empoderamiento de los trabajadores. Este modelo de relaciones se sostiene en un sistema normativo cuyas bases no se han modificado sustantivamente desde el Plan Laboral implementado durante el régimen militar, y que impone una serie de restricciones a los derechos laborales de los trabajadores, en especial los de indole colectiva. La permanencia de este modelo se expresa en un debilitamiento creciente del sindicalismo y la defensa de los derechos colectivos desde el reestablecimiento de la democracia. ${ }^{22}$ En este contexto, las mujeres están más expuestas a situaciones de violencia que sus compañeros (Ansoleaga et al 2016). ${ }^{8}$

\section{Violencia de género en el trabajo}

Desde la perspectiva de la hipótesis del desbalance de poder entre víctimas y victimarios, las mujeres están doblemente expuestas a violencia laboral por un bajo poder formal en la estructura ocupacional y por un bajo poder social que emana de relaciones de género que subordinan y discrimina a las mujeres.

\section{Violencia asociada a la posición de las mujeres en la estructura ocupacional}

El género es una dimensión determinante de la menor participación laboral de las mujeres, de su acceso a un limitado abanico de ocupaciones, de sus menores salarios y de las dificultades que tienen para acceder a posiciones de poder en las organizaciones así como en otros espacios sociales. Por el contrario, los hombres acceden a un espectro mucho más amplio de ocupaciones, predominan en trabajos mejor pagados y de mayor estatus, ocupando con mayor frecuencia que las mujeres posiciones de jefaturas y supervisión. ${ }^{23}$

En los sectores que se realizó el estudio la información disponible permite ilustrar que, a pesar del crecimiento del país y del aumento de la participación laboral femenina, en términos de calidad del empleo la situación de las mujeres es desventajosa respecto de sus pares hombres.

El retail es una importante industria para el empleo femenino. El año 2010 empleaba a 582 mil asalariados del sector privado, de los cuales el 51,1\% correspondía a mujeres, cifra que aumentó a un $54,8 \%$ en el 2013. Si bien el empleo asalariado de este sector creció en solo un 0,8\% entre 2010 y 2013, en el caso de las mujeres creció en un 8,1\%. Respecto a las condiciones de empleo, el sector muestra una precariedad laboral extendida que afecta más a las mujeres. Por ejemplo en el período señalado aumentaron los contratos a plazo fijo a un $20 \%$ no así entre los hombres y se mantuvo una importante brecha salarial de género contra las mujeres. ${ }^{19}$ La precariedad contractual se asocia a inestabilidad laboral y temor al despido, lo cual aumenta la tolerancia a arbitrariedades y maltrato y reduce la capacidad de afrontamiento.

“(...) la gente anda muy atemorizada, sobre todo lo que es la mujer, ¿por qué? porque se sienten desprotegidas porque saben que ¿si me echan de aqui qué hago? entonces pasa eso mucho" (Dirigente sindical, industria del retail)

Debido al temor de perder el empleo se aceptan cambios arbitrarios de las condiciones de trabajo: "Yo soy vendedora, y mi jefe me explota, me hace quedarme continuamente hasta muy tarde, antes éramos 4 ahora somos 2 y no han traídos reemplazos, tengo que hacer yo la pega que antes hacíamos varios.... un jefe estaba diciendo que a los que no trabajaran los sobre tiempo que les pedian los iba a echar a todas, entonces asustadas por esas cosas te fijas?" (Trabajadora, industria del retail).

En la industria bancaria, el empleo de las mujeres creció en un 44,6\% versus un 19,3\% de crecimiento de los hombres. Si bien, el sector se caracteriza por una alta formalidad en sus trabajadores/as directos, ha incrementado en gran medida el empleo externalizado, especialmente el empleo femenino, y por la práctica cada vez más extendida de aplicar remuneraciones según rendimiento y cumplimiento de metas como medio de intensificación del trabajo. ${ }^{19}$ Esta crea ambientes propicios para la competencia y el hostigamiento laboral de las jefaturas, en particular a las mujeres que son la fuerza de ventas del sector financiero.

"Yo creo que los niveles a los cuales se les puede extraer mayor cuota de renta, de plusvalor, son los sectores más dinámicos del área de las finanzas. Esos sectores están ubicados estratégicamente, son los que colocan créditos, los que venden dinero, es el área operativa que tiene que dar el sustento para que eso se materialice en la organicidad de las instituciones. Las fuerzas de venta hoy dia que tienen los bancos para colocar y aumentar las colocaciones son mayoritariamente personal femenino, 90 y tanto por ciento son mujeres. Las áreas denominadas call center que son los centros de llamados que tienen los bancos que son filiales de los bancos, sociedades de apoyo al giro, bueno esos también están conformadas por una cantidad impresionante de mujeres. Bueno ese sector es el que quizás sufre con mayor sistematización el tema de la violencia. (Dirigente sindical, industria bancaria). Otro dirigente de la industria bancaria reitera el testimonio anterior señalando que "un gerente insultaba a garabatos a las colocadoras de productos "tales por cuales tienen que cumplir con las metas si no se van cagando del banco weonas flojas..."

La industria de alimentos también ha absorbido fuerza de trabajo femenina, siendo en la actualidad el 34,4\% del total empleado en el sector. Entre 2010 y 2013 el empleo mostró un crecimiento total de14\% y de un 39\% para las mujeres. La calidad del empleo femenino muestra mayores rasgos de precariedad que el empleo masculino. Un 29\% de las mujeres tiene contrato a plazo fijo versus un 20\% de los hombres, y un 14\% de mujeres trabaja sin contrato (versus 11\% de hombres). Así también, el empleo asalariado externo femenino ha mostrado una tendencia al crecimiento en las mujeres en un 22\% entre 2010 y 2013. ${ }^{19}$ Estas condiciones generan inestabilidad laboral que, como se ha visto en otras situaciones, determina una nula capacidad de enfrentamiento de las mujeres ante el maltrato.

"Yo siempre digo 'si no estuvieran los 2 sindicatos en esta empresa a lo mejor la realidad y el escenario sería muy distintos con los trabajadores y en especial con las mujeres porque el hombre tiende más a reaccionar, a no permitir, pero en el caso de las mujeres que vienen, como decian las mismas muchachas de trabajos anteriores a este, de temporera, aqui hay mucha mujer que trabajaba de temporera, 7 meses de trabajo y el resto nada y con ingresos mucho menores, entonces valoran más estar hoy día aqui en la empresa, y ese valorar más y la necesidad que tienen de repente son las jefas 
de hogar hacen que ellas mismas permitan más que los hombres un maltrato" (Dirigente sindical, industria de alimentos).

$\mathrm{Al}$ igual que en otros sectores, en la industria de alimentos los sueldos se componen de una parte fija pequeña y de una parte variable determinada por el cumplimiento de metas de producción lo cual es una fuente de conflictos entre compañeros/as de trabajo, particularmente cuando los incentivos son colectivos.

Una trabajadora relata que: “...hay momentos en que estamos en buena, pero hay momentos en que estamos colapsadas, que no quiero que ni mi compañera me hable, me pasa que estoy trabajando a full, todas trabajando y me busca alguien en la puerta, yo no quería salir porque estaba apurada, quería sacar mi trabajo y le contesté mal, ¿me entiende? Y si yo paro de hacer esto, y voy a ver una cosa que no corresponde al trabajo mis compañeras murmuran y dicen: claro, fue a atender allá, claro, y le estamos haciendo la pega, y empiezan 'para que te quería' y empieza la cosa, empieza, el ambiente ¿y para esa huevada te querían? y empieza el ambiente ¿entiende? Te estamos haciendo el sueldo... Entonces... todo es generado ahi” (Trabajadora, industria de alimentos).

En los tres sectores, los cargos de jefaturas más altos son ocupados mayoritariamente por hombres. En el retail, 1,2 \% de los hombres versus in $0,4 \%$ de las mujeres ocupan cargos directivos; en el sector de intermediación financiera lo hace 5,2\% de los hombres versus un 2,6\% de mujeres, y en la industria de alimentos, un 1,7\% de los hombres versus un $0,4 \%$ de las mujeres ocupan cargos directivos.

En palabras de un dirigente sindical del retail: "El personal casi en un 100\% son mujeres, sin embargo, los supervisores son hombres, curiosamente..."

\section{Violencia asociada a desigualdades y discriminaciones de género}

De acuerdo a los resultados de este estudio, la violencia laboral hacia las mujeres se expresa en los espacios de trabajo en actitudes y conductas "machistas", en acoso sexual y en discriminaciones asociadas al uso de derechos de maternidad.

- Acoso por actitudes y conductas "machistas"

Muchas mujeres, en sus testimonios, señalan que son víctimas de hostigamiento por parte de los hombres que integran el personal de la empresa en la que trabajan. Se refieren a "los hombres, los varones" sin diferenciar, a menudo, si son compañeros o jefes, reconociendo con ello que sus comportamientos se asocian a una cultura machista prevaleciente en las interacciones sociales generales y que rebasan las estructuras de poder formal de las empresas. “(...) lamentablemente las mujeres sufrimos harto mobbing, por parte de los varones. Es una sociedad bastante machista y que se traspasa a la empresa y que no es en definitiva el área laboral la que lo produce" (Dirigenta sindical, industria bancaria)

"(los compañeros) andan constantemente piropeando y no se dan cuenta que eso también es un acoso" (Trabajadora, industria del retail)

El problema del machismo como problema cultural más amplio que traspasa los límites de la empresa es destacado también por dirigentes hombres.

"Mira yo creo que la ley avanzó mucho cuando se dijo vamos a llevar la ciudadanía dentro del trabajo, con la tutela del derecho fundamental etc. etc. Pero lo primero que llegó dentro de las empresas eran todas las malas prácticas sociales, entre ellas por supuesto, el machismo ¿por qué? porque ya trasciende el problema laboral, yo creo que es un tema cultural y que en el ámbito de la empresa donde se da, es porque se está igual que en la casa entre cuatro paredes, se abusa, ese es mi concepto, en ese sentido lo observo" (Dirigente sindical, industria de alimentos)

Las expresiones de machismo se manifiestan con cierta frecuencia en conductas discriminatorias menos visibles que otras formas abiertas de agresión. Una dirigenta sindical de la banca señala, por ejemplo, que "los varones muchas veces se sienten tal vez como desplazados por una mujer y a uno la atacan ya sea que puede ser un acoso sexual, o hablando de temas que a las mujeres no nos gusta, en forma vulgar muchas veces, entonces uno se siente incómoda, eso también es una forma de agresividad".

Asimismo, una joven que ocupa un cargo importante de jefatura en una industria de alimentos relata que es discriminada por sus colegas, situación que ella atribuye a tres condiciones que definen su identidad: ser mujer, joven y haber adquirido un alto nivel de calificación profesional fuera del país. De acuerdo a Cortina ${ }^{24}$ muchas de estas manifestaciones encajan con el concepto de incivismo selectivo en el lugar de trabajo, que afecta más probablemente a mujeres y minorías exitosas profesional y económicamente que son, por esto, percibidas como altamente competentes por los hombres en un campo dominado por ellos lo que les genera sentimientos de amenaza, competencia, y hostilidad hacia miembros de ese grupo externo que invade su territorio.

"Yo soy mucho más joven que todos los otros (...) dentro de la línea como de gerentes, yo soy 10 años más joven que cualquiera, entonces yo tengo más resistencia o tuve más resistencia de mis compañeros, de mis pares que del equipo que dirijo. En general yo siento más como una discriminación por decirlo de alguna forma por género o por edad, muy fuerte de mis pares. Son todos hombres y en su mayoría, yo diría de partida por edad son 10 años mayores que yo y por años de experiencia en la compañia todos tienen 15 años, 20 años y yo llevo 6 años en la compañia y tengo 10 años menos que ellos, entonces (...) yo soy la que tiene que salir a demostrarles (mi capacidad). Bueno, de partida hacen reuniones de gerentes, porque yo estoy de gerente y ahi es donde unos dicen 'no pero usted no es gerente' y tenemos el mismo grado, y de mi depende más gente, entonces muchas veces me dejan afuera" (Mujer en cargo de Jefatura, industria de alimentos).

Lo que parecen expresar estos dos casos es que, aún cuando las mujeres adquieran mayor poder formal que sus colegas, estos ejercen sobre ellas el poder social que emana de la masculinidad, mediante agresiones veladas o discriminaciones.

Cabe destacar que las representaciones sociales de género se manifiestan también en comportamientos de mal trato de parte de clientas y clientas hacia mujeres que trabajan en atención a público. De acuerdo a sus testimonios, se les atribuye a ellas menor capacidad de respuesta y afrontamiento ante situaciones de violencia, versus la percepción de que los hombres pueden responder a esos comportamientos, y a veces, incluso agresivamente.

“(...) el cliente fuera de control, con el hombre no se pasa mucho de revoluciones, pero con la mujer le larga todo, se le pasa la mano con las mujeres, vendedoras, asesoras e incluso mujeres reclamando en forma descontrolada también se van contra las mujeres, porque temen que enfrentada a un vendedor, este las mande a la puta madre. Yo creo que porque las ven más vulnerables, las ven más débiles y le tiran el camión encima fundamentalmente en áreas como servicio al cliente, ocurre mucho, todos los dias las putean, esa gente tiene que tener sangre de horchata" (Hombre en cargo de Jefatura, industria del retail). 


\section{- Acoso sexual}

El acoso sexual constituye una de las expresiones más evidentes de la distribución asimétrica y jerárquica del poder entre hombres y mujeres en la sociedad. Fue tipificado y sancionado en la normativa laboral chilena en la Ley 20.005 del año 2005, la cual estableció que se produce acoso sexual cuando una persona realiza en forma indebida, por cualquier medio, requerimientos de carácter sexual no consentidos por quien los recibe y que amenazan o perjudican su situación laboral o sus oportunidades en el empleo. La Ley establece asimismo que las empresas deben tener un reglamento interno que fija procedimientos para la investigación y sanción del acoso sexual en el trabajo.

Se distinguen dos tipos básicos de acoso sexual. El acoso quid pro quo y el que crea un ambiente de trabajo hostil. El primero es propiamente un chantaje sexual por el cual se fuerza a una trabajadora (o trabajador) a elegir entre someterse a los requerimientos sexuales o perder o ver perjudicados ciertos beneficios o condiciones del trabajo. La segunda forma de acoso sexual es la que crea un entorno laboral intimidatorio, hostil o humillante para el/la destinatario/a. ${ }^{25}$

Ilustran la primera situación los siguientes testimonios:

"Más que nada el problema de nosotros es el acoso sexual, lo demás todo es manejable. Hemos hablado el tema, lo hemos revisado pero nos falta alguien que se atreva de verdad a decirlo... Yo creo que la mayoría es por miedo a lo que le puedan hacer, decir, que las puedan cambiar de tienda, esa es como la pesadilla que tienen las chicas y quieren mantenerse piola sin crear conflictos, tratan de mantenerse y dicen no puedo (denunciar)." (Dirigente sindical, industria del retail).

"En una oportunidad en un local habia una niña que era muy estupenda, y disculpe la palabra que voy a usar, dice 'ya viene este viejo desgraciado, me amenazó me dijo que si no le daba la pasada me tenia que ir" (Trabajador, industria del retail).

Sin embargo, en la práctica es difícil distinguir una forma de otra. Por una parte, las trabajadoras saben que la no aceptación de requerimientos sexuales encierra la amenaza de despido o de pérdida de beneficios, aunque esta no sea explícita. Por otra parte, estos requerimientos generan sentimientos de humillación, vergüenza y confusión. Asimismo, el rechazo puede conducir a otras formas de hostigamiento lo cual podría explicar la mayor prevalencia de acoso psicológico de las mujeres en los espacios de trabajo. Para evitar las consecuencias que pueden seguir a una denuncia de acoso sexual o de otra forma de hostigamiento, o bien, para ocultar la humillación y sentimiento de culpa ante un medio social que suele culparlas, muchas mujeres soportan estos requerimientos en silencio.

"(Las niñas) no dicen nada, se callan, hay muchas que se quedan calladas, es que da vergüenza yo creo, da vergüenza, y se quedan calladas la mayoría". (Trabajadora, industria de alimentos).

Un dirigente destaca que aún existiendo en las empresas políticas explícitas para evitar acoso sexual, en la práctica, se culpabiliza a las trabajadoras que son quienes terminan siendo removidas de sus trabajos.

"Si, hay politicas explícitas pero las veces que han operado, en general la persona que fue violentada es la que sale, claro la que mueven, salvo que ya tenga muchas denuncias a su haber la persona que es el acosador, ahi puede ser..." (Dirigenta sindical, industria bancaria).

Lo que posibilita el acoso sexual en el trabajo es la concentración total del poder en manos de un jefe varón de quien depende una mujer para tener o conservar un empleo y que determine su sueldo, su evaluación o posibilidades de ascenso. ${ }^{18}$

"Si, yo he visto, no me han contado, de algunos jefes, que persiguen a las trabajadoras para el baño y las esperan... (Ellas) se ponian a llorar en el baño porque el viejo estaba afuera, afuera esperándolas." (Trabajadora, industria de alimentos).

Esta percepción de las trabajadoras es respaldada por información de la Dirección del Trabajo que reporta 302 denuncias de acoso sexual recibidas el año 2008, de las cuales en el 80,5\% de los casos las personas denunciadas son superiores jerárquicos (gerentes, jefaturas o representantes de la empresa) es decir, la denuncia de acoso sexual está vinculada al abuso de poder jerárquico formal. ${ }^{2}$

\section{- Violencia de género por maternidad}

En las últimas décadas las mujeres chilenas han ingresado crecientemente al mercado de trabajo, proceso que ha generado fuertes tensiones derivadas de la persistencia de un modelo de familia y de relaciones de género que asigna a las mujeres la responsabilidad del trabajo reproductivo -trabajo doméstico y cuidado no remunerado-. "Aunque el discurso social da a la maternidad un rol básico en la sociedad, en el desempeño laboral la función materna y el rol de cuidadora de los hijos menores son vistos como una interferencia negativa" 26 , fundamentalmente por el mito de que la maternidad aumentaría los costos laborales, el ausentismo y la rotación laboral de las mujeres, lo cual incidiría, a su vez, en su productividad. No obstante, un importante estudio de la Oficina Regional para América Latina de la OIT, realizado en cinco países (Chile, Brasil, Argentina, Uruguay y México) sobre costos laborales de hombres y mujeres desmiente este supuesto, demostrando que "los costos monetarios directos para el/a empleador/a asociados a la protección de la maternidad y al cuidado infantil son muy reducidos, representando en los cinco países analizados menos del 2\% de la remuneración bruta mensual de las mujeres". ${ }^{27}$

A pesar de estos antecedentes, los y las entrevistadas/os dan cuenta de situaciones de maltrato, y discriminaciones a las mujeres que se expresan en vulneración de derechos de maternidad, amenazas de despido y de limitarles los ascensos en sus lugares de trabajo a quienes se embarazan y tienen hijos.

Un hombre dirigente sindical de la industria bancaria, ilustra la forma cómo se manifiestan esas situaciones en las empresas del sector.

“(...) el hecho del embarazo, de la maternidad, en la banca es una práctica que cada vez adquiere una relevancia mayor y que suena irónicamente porque justamente en la banca y en el sistema financiero hay instalado un discurso permanente del derecho a la vida y el derecho a la maternidad, pero sin embargo en la práctica común, corriente, eso es solamente un discurso, en los hechos concretos se está presionando a la mujer para que no sea madre, para que no pueda ejercitar este derecho tan fundamental que está garantizado en la constitución y en las leyes...”. (Dirigente sindical, industria bancaria).

Asimismo, una mujer dirigenta del mismo sector, describe las presiones para que las mujeres renuncien a derechos de la maternidad, como el derecho a alimentación e incluso al permiso prenatal.

"En realidad para la mujer es bien difícil el área laboral, porque tiene que sortear bastantes dificultades, existe una violencia muchas veces solapada, también hay una violencia cuando uno es madre cuando uno quiere acceder a los derechos de la maternidad 
como la alimentación para su hijo menor. Muchas veces ahi ya empieza una violencia. A mi me tocó personalmente, yo tengo 4 hijos, cuando yo volvía de mis embarazos en la empresa me sacaban de mi cargo y me ponian en un pool que es un cargo que uno va rotando en todas las sucursales de Santiago, y eso lo hacian durante el periodo de fuero y los 2 años que duraba el derecho de de alimentación para el niño menor. Entonces uno se siente maltratada. Después también muchas veces los jefes no dejaban que uno se tomara este tiempo aduciendo que iba a tener menor productividad. Hay muchas mujeres que no se toman estos derechos. En la experiencia de las socias, incluso hay un caso dramático de una socia que ya tenía 3 hijos no contó que estaba embarazada de su cuarto hijo, y no lo contó hasta que un día en el trabajo se sintió mal y fue a dar a luz a su hijo, o sea es un caso bastante dramático". (Dirigenta sindical, industria bancaria).

Ella continúa su testimonio señalando las limitaciones de desarrollo profesional asociadas al hecho de ser madres.

“(...) también no son bien consideradas, tienen pocas expectativas de desarrollarse en la empresa una vez que son madres; las mujeres que se desarrollan más son las solteras sin hijos, pero una vez que ya tuvieron hijo ya la empresa ya no las toma tanto en cuenta". Testimonios similares fueron entregados por trabajadoras de la industria del retail.

"Bueno, el tema de xxxx (Supermercado) es que una mujer embarazada es un cacho, ¿por qué? porque tienen que tener privilegios, darles permiso para ir al médico, a controles, entonces a la empresa no le sirve ese tipo de mujeres, cada vez que alguien está embarazada 'ah ya, un cacho más'. Entonces no tenemos ese privilegio. Ahora también, en cuanto a los ascensos también son discriminadas las mujeres que tienen niños pequeños y se los dicen literalmente: ¿sabes qué? tú no estás apta porque necesitamos una persona al 100\%' y lamentablemente es mamá y no puede”. (Trabajadora, industria del retail).

También hay ejecutivos conscientes de esta tensión y no comparten las actitudes empresariales frente a la maternidad. En la industria de alimentos un ejecutivo relata:

"Habia un jefe que las amenazaba si se quedaban embarazadas, o sea si recuerdo 'pobre de la que se embarace'. Yo en ese momento era parte de un equipo de mucha gente entonces yo era nuevo entonces encontraba que era un poco chocante, pero no tienes ni la autoridad ni la influencia como para poder cambiar eso". (Hombre en cargo de jefatura, industria de alimentos).

Otros estudios en Chile han revelado también que mujeres trabajadoras advierten acerca de la fuerte resistencia de parte de los empresarios a contratar mujeres por lo que ellas señalan como "el problema de la maternidad" y que, a pesar de reconocer que existe una mayor tolerancia respecto a la contratación de mujeres, persiste en el empresariado la percepción de que la maternidad es un obstáculo para el desempeño laboral lo cual, a su vez, justificaría sus bajos salarios: "lo que pasa es que se te limita trabajar por la maternidad... por eso veo que la mujer ¿qué está haciendo ahora? está postergando su maternidad, o te van a pagar un minimo...". ${ }^{28}$

Riquelme señala que el conflicto entre maternidad y trabajo supone dos momentos críticos para las empresas y las trabajadoras. El primero ocurre al comunicar el embarazo a la empresa, lo que genera una serie de consecuencias negativas para las mujeres: el 70,5\% del total de denuncias por discriminación por maternidad son realizadas en ese momento; mientras que el 29,5\% restante se produce al regreso de la licencia de postnatal.
Las trabajadoras denuncian reiteradamente que son despedidas luego de comunicar su embarazo al empleador, pese al fuero que las protege. Por otra parte, un número significativo de las denunciantes señala que la reincorporación de la trabajadora por la Inspección del Trabajo es, muchas veces, el detonante de los hostigamientos y malos tratos. ${ }^{26}$

Piñuel $^{29}$ llama a este problema "mobbing maternal", aludiendo con ello a la violencia o acoso que sufre la mujer por el hecho de ser madre, especialmente en su entorno laboral. No se busca la destrucción de la víctima de forma directa, sino que lo que se busca más inmediata y directamente es mostrar un ejemplo de lo que le puede pasar a "la que se atreva a..." quedarse embarazada. Es un castigo ejemplar que sirva de aviso para otras mujeres que observan lo que le ocurre a la víctima, y no se atrevan a ejercer su derecho a la maternidad. En síntesis, lo que se busca es evitar nuevos embarazos en la empresa

\section{CONCLUSIONES}

De acuerdo a las percepciones de las personas entrevistadas en este estudio, en especial trabajadores/as y dirigentes/as sindicales, los resultados más importantes a destacar son:

i. Que la dimensión más determinante de la violencia en el trabajo es el predominio de un sistema de relaciones laborales extremadamente vertical, y de estilos de liderazgos, de supervisión y control autoritarios que se ejercen mediante una amplia gama de formas de violencia, que es a la vez, sostenida y dirigida al colectivo de trabajadores/as, respondiendo a lo que se ha conceptualizado como "régimen de trabajo opresivo".

- Que este sistema se sostiene en un desbalance de poder entre trabajadores/as y capital, considerado este el determinante más importante de la violencia por la débil capacidad de afrontamiento de quienes reciben las agresiones: los y las trabajadoras.

- La ausencia de respuestas colectivas a la violencia vertical puede dar lugar a violencia entre pares causadas por condiciones de trabajo que los/as trabajadores/as no pueden modificar por el bajo poder de negociación en las organizaciones, por ejemplo los bonos colectivos por productividad, o exigencia de metas excesivamente altas, entre otras.

- En este contexto, las mujeres están doblemente expuestas a violencia laboral por un bajo poder formal en la estructura ocupacional y por un bajo poder social que emana de relaciones de género que las subordinan y discriminan. Se expresa en violencia asociada a condiciones precarias de empleo, fundamentalmente en amenazas de despido o de cambio de sus condiciones de empleo; en diversas formas de violencia derivadas de la prevalencia en los espacios de trabajo de una cultura machista; específicamente en acoso sexual y en discriminaciones y acoso por maternidad. El rechazo o la denuncia de estas situaciones implica a menudo el despido de las trabajadoras -no del acosador- o detona otras formas de hostigamiento y maltrato.

- La violencia de género que se produce en el ámbito doméstico ha constituido un foco central de atención del Estado de Chile por su creciente magnitud e impacto. Para abordar la violencia contra las mujeres, ha ratificado instrumentos del sistema internacional de derechos humanos comprometiéndose a erradicar la violencia contra las mujeres y ha implementado 
además un amplio marco jurídico legal para abordarla a nivel nacional. Sin embargo tanto el marco legal chileno como el abordaje de las políticas no apuntan central ni ampliamente hacia todas las formas de violencia que se ejerce contra las mujeres focalizándose principalmente en la violencia doméstica. $^{30}$ La violencia en el trabajo, ha en general sido poco abordada, como hemos señalado anteriormente, pero la violencia de género en el trabajo lo ha sido menos aún. No es posible comprender los determinantes de la violencia de género en el trabajo bajo el supuesto de que las organizaciones son entidades neutrales. El ámbito del trabajo es un espacio de reproducción del sistema de relaciones sociales en un sentido amplio, y en particular, del sistema de relaciones de género que desvaloriza y discrimina a las mujeres.

Esta investigación fue financiada por Fondecyt, Concurso Regular 2014, Proyecto No 1140060.

Fue realizada por el Programa de Estudios Psicosociales del Trabajo de la UDP y el Centro de Estudios de la Mujer.

\section{REFERENCIAS}

1. Chapelle D, Di Martino V. Violence at work. 3rd ed. Ginebra: International Labour Office; 2006.

2. Dirección del Trabajo. Acoso sexual en el trabajo ¿Denunciar o sufrir en silencio? Análisis de Denuncias. Santiago de Chile: Dirección del TrabajoMinisterio del Trabajo y Previsión Social; 2009. (Aporte al debate, 23).

3. Castellón D. Violencia laboral en enfermeras: explicaciones y estrategias de afrontamiento. Rev Latino-Am Enfermagem. 2011;19(1):153-63.

4. Letelier $\mathrm{P}$, Valenzuela $\mathrm{S}$. Violencia: fenómeno relevante de estudio en campos clínicos intra-hospitalarios de enfermeria. Cienc enferm. 2002; 8(2):13-19.

5. Paravic T, Valenzuela S, Burgos M. Violencia Percibida por Trabajadores de Atención Primaria de Salud. Cienc. enferm. 2004;10(2):53-65.

6. Paredes Arévalo L, Paravic Klijn T. Percepción de violencia y factores asociados según usuarios de un consultorio y postas de salud. Concepción, Chile. Cienc enferm. 2006; 12(1):39-51.

7. Macia $F_{1}$ Reynaldos $C$. Acoso psicológico laboral: Nociones generales. Su Presencia y estudio en Chile. Santiago de Chile: RIPSOL-UDP; 2009.

8. Ansoleaga $E$, Díaz $X$, Mauro A. Gendered work violence issues and mental health among Chilean women workers. En: Gideon J, editora. Handbook on Gender and Health. London: Edward Edgar Publishing; 2016.

9. Einarsen $\mathrm{S}$, Rakne, BI, Matthiesen SB. Bullying and harassment at work and their relationship to work environment quality: An exploratory study. Eur J Work Organ Psych. 1994; 4(4):381-401.

10. Hoel $H_{1}$ Beale D. Workplace bullying, psychological perspective and industrial relations: toward a contextualized and interdisciplinary approach. $\mathrm{Br} \mathrm{J}$ Ind Relat. 2006; 44:2:239-262.

11. Salin D. Ways of explaining workplace bullying. A review of enabling, motivating, and precipitating structures and processes in the work environment. Hum Relat. 2003;56(10): 1213-1232.

12. Beal D. An industrial relations perspective of workplace Bullying. In: Einarsen E, Hoel H, Zapf D, Cooper CL, editores. Bullying and Harassment in the workplace. Developments in Theory, Research and Practice. 2nd ed. London: CRC Press; 2011. p. 283-300

13. Ironside $M$, Seifert R. Tackling bullying in the workplacae. The collective dimension. In: Einarsen $\mathrm{S}$, Hoel $\mathrm{H}$, Zapf $\mathrm{D}$, Cooper $\mathrm{CL}$, editores. Bullying and emotional abuse in the workplace: International perspectives in research and practice. London: Taylor \& Francis; 2003. p. 383-398.

14. Einarsen $S$, Hoel $H$, Zapf $D$, Cooper $C L$. The concept of bullying and harassmente at work. The European Tradition. In: Einarsen S, Hoel H, Zapf D, Cooper CL, editores. Bullying and Harassment in the Workplace. Developments in Theory, Research, and Practice. London: CRC Press; 2011. p. 3-40.

15. Cortina L, Magley V, Hunter J, Day Langhout R. Incivility in the workplace: incident and impact. J Occup Health Psychol. 2001; 6(1):64-80.

16. Espino, Alma. Género e investigación en ciencias sociales y economía. Economía y Sociedad (Lima); 2006; 61:7-14.

17.- Sen $G$, George $A$, Östlin P. Incorporar la perspectiva de género en la equidad en salud: un análisis de la investigación y de las políticas. Washington, D.C: OPS-Harvard Center for Population and Development Studies; 2005.
18. Rico N. Violencia de Género. Un problema de Derechos Humanos. Santiago de Chile: CEPAL; 1996. (Mujer y Desarrollo, 16).

19. Universidad Diego Portales-CEM. Industrias de Elaboración de Alimentos y Bebidas, Retail y Sector Financiero, Caracteristicas Económicas, del Empleo y de la Situación Sindical por sector (no publicado). Santiago de Chile: UDP; 2014.

20. Ramos Zincke C. La modernización de la empresa chilena: posfordismo con huellas autoritarias. En: Stecher A, Godoy L, editores. Transformaciones del trabajo, subjetividades e identidades. Lecturas psicosociales desde Chile y América Latina. Santiago de Chile: RIL; 2014.

21. Rodriguez, J, Gómez C.F. HRM in Chile: the impact of organizational culture. Employee Relat. 2009; 31(3):276-294.

22. Universidad Diego Portales. Informe XIII Derechos Humanos. Santiago de Chile: UDP; 2010.

23. Cruz A, Klinger S. Gender-based violence in the world of work: Overview and selected annotated bibliography. Geneva: International Labour Office; 2011. (Working Paper, 3/11).

24. Cortina L. Unseen injustice: incivility as modern discrimination in organizations. Acad Manage Rev. 2008; 33(1):55-75.

25. Organización Internacional del Trabajo-OIT. Acoso sexual en el trabajo y masculinidad. Exploración con hombres de la población general. Centroamérica y República Dominicana. San José: OIT; 2013.

26. Riquelme V. ¿La Maternidad Castigada? Discriminación y Malos tratos. Santiago de Chile: Dirección del Trabajo-Ministerio del Trabajo y Previsión Social; 2011. (Aporte al Debate, 25).

27. Abramo L, Todaro R. Costos laborales y reproducción social: Análisis comparativo de cinco paises latinoamericanos. En: Abramo L, Todaro R. editoras. Cuestionando un mito: Costos laborales de hombres y mujeres en América Latina. Lima: OIT, Oficina Regional para América Latina y el Caribe; 2002. p.11-50.

28. Díaz $X$, Mauro A, Todaro R. Restricciones al empoderamiento económico: la mirada de las mujeres. El caso chileno. Santiago de Chile: IDRC/CIEDUR/ CEDLA/ONU Mujeres/CEM. En prensa 2016.

29. Piñuel I. Mobbing maternal: el acoso psicológico contra las mujeres embarazadas. Málaga: UAH; 2009 [en línea] [consultado dic 2016]. Disponible en: http:// acosopsicologico.blogspot.com/2009/09/mobbing-maternalel-acoso-psicologico.html

30. Observatorio de Equidad de Género en Salud-OEGS. Informe Monográfico 2007-2012. Violencia de Género en Chile. Santiago de Chile: OPS; 2013.

\section{Referencias complementarias}

Alvesson $M$, Billing, YD. Understanding Gender and Organization. London: SAGE Publications; 1997.

Krieger N, Waterman D, Hartman C, Bates L, Stoddard A, Quinn M, Sorensen G, Barbea E. Social hazards on the job: workplace abuse, sexual harassment, and racial discrimination. A study of black, latino, and white low-income women and men workers in the United States. Int J Health Serv. 2006; 36(1):51-85. 